KocHER, Gernot

DOI: 10.15170/DIKE.2020.04.01.07

Em. o. Prof. Dr. iur. Dr. h. c.

Karl-Franzens-Universität Graz

\title{
Krieg und Frieden - Aspekte der Vielseitigkeit von Sprache und Bild im Recht
}

\section{War and Peace - Aspects of Versatility of Language and Picture in Law}

The aim of the study is to compare linguistic and visual expressiveness. The study deals with the words war and peace, which have a complex meaning. The ckeck is carried out on hand of five illustrations of the catalan Vidal Mayor (around 1300), which actually make it clear that there is also a variety of ways of expressing the conditions of war and peace in the image area.

Keywords: iconography of law, legal language, war, peace, litigation, signs in court

\section{Vorwort}

Die wissenschaftliche und im Laufe der Zeit auch freundschaftlich gewordene Beziehung zu István Kajtár reicht bis in die Siebzigerjahre des vorigen Jahrhunderts zurück, als ich im Rahmen der Partnerschaft unserer Universitäten im wissenschaftlichen Austausch in Pécs war. Als aus dieser doch eher lockeren Partnerschaft eine enge rechtsgeschichtliche Kooperation wurde, ${ }^{1}$ war einer der Schwerpunkte, der uns beide faszinierte, der Bereich Bild und Recht. Deshalb folgt auch dieser Beitrag zum Gedächtnis an István Kajtár dieser gemeinsamen Interessenlinie; zusätzlich trägt auch die Ausrichtung auf „Krieg und Frieden“ im weitesten Sinne seiner speziellen Vorliebe für die Seekriegsführung Rechnung.

\section{Der rechtssprachliche Ausgangspunkt}

Die rechtssprachliche Bandbreite von „Krieg“ und „Frieden“ ist durch die moderne Entwicklung (insbesondere durch das Völkerrecht) in die Richtung - meist zwischenstaatlicher - militärischer Auseinandersetzung verschoben und damit auch eingeschränkt worden. Das gilt auch teilweise für die Rechtsgeschichte: So verortet der Artikel „Krieg“ in der zweiten Auflage ${ }^{2}$ des Handwörterbuches zur Deutschen Rechtsgeschichte den Begriff überwiegend im militärischen Bereich, während die Bezeichnung „Krieg“ für privat- oder strafrechtlich relevantes Handeln nur minimal am Rande gestreift wird. Der Weiterverweis von „Kriegsbefestigung“ auf litis contestatio ${ }^{3}$

\footnotetext{
${ }^{1}$ Vgl. dazu Herger - Korsósné DeLACASSE, Recht ohne Grenzen und KajTÁr, Bevezetés a jogi kultúrtörténetbe (= Einführung in die rechtliche Kulturgeschichte).

${ }^{2}$ MÜNKLER, Krieg 22-247; die erste Auflage enthält dieses Stichwort gar nicht.

${ }^{3}$ CORDES - HAFERKAMP - LÜCK, Handwörterbuch 251.
} 
ist ebenfalls nur als kleiner Hinweis auf die ursprünglich weite Bedeutung des Wortes „Krieg“ zu verstehen. Dabei hätte der einfache Verweis auf das Deutschen Rechtswörterbuch ${ }^{4}$ mit seiner klaren Differenzierung zwischen „Krieg“ als Auseinandersetzung im „rechtsförmlichen Verfahren“棌 und „Krieg“ als „bewaffnete Auseinandersetzung zwischen Staaten“ mit den dazu gebotenen umfangreichen sprachlichen Variationsformen den nach einer Erklärung suchenden Leser doch leicht auf diesen zweiten, historisch wichtigen Bedeutungsstrang bringen können.

Besser steht es um den „Frieden“: Als „Hochwertwort“ im Handwörterbuchartikel ${ }^{6}$ eingestuft, wird dessen Bedeutungsbreite auch dementsprechend gewürdigt; der Artikel entspricht damit auch der Bedeutungsfülle der Belege im Rechtswörterbuch, wobei die speziellen Untergruppen „III. Institutionalisierungen“7 und „V. Pax et iustitia“ ${ }^{\text {“8 }}$ im Hinblick auf die folgenden Visualisierungbeispiele mit ihrer Konzentration auf den König als Friedenswahrer beziehungsweisen das Recht als Garant des Friedens aufschlußreich sind.

\section{Der visuelle Blickwinkel}

Die bildverwertende rechtsgeschichtliche Forschung in unterschiedlicher Intensität gibt es im deutschsprachigen Raum seit fast zweihundertfünfzig Jahren, ${ }^{9}$ allerdings mit einer Konzentration auf die vier illustrierten Handschriften des Sachsenspiegels (Codices picturati), denn rechtsikonographische Konzeption und Realisierung sind einmalig. Die anderen mittelalterlichen rechtlichen Bildquellen ${ }^{10}$ sind dagegen in den Hintergrund getreten, wohl weil ihnen das fortlaufende Illustrationskonzept der Codices picturati fehlt, jedoch in Mißachtung der Tatsache, dass auch abschnittsbezogene Einzelillustrationen enorm aussagekräftig sein können. ${ }^{11}$ In diesen Kreis der „anderen mittelalterlichen Bildquellen“ gehört auch eine illustrierte Rechtshandschrift katalanischen Ursprungs, der sogenannte Vidal Mayor aus der zweiten Hälfte des 13. Jahrhunderts. Der Sammlerzufall hat sie nach Deutschland gebracht und da ist sie in das Visier romanistischer ${ }^{12}$ und kunsthistorischer Forschung ${ }^{13}$ geraten, während der beachtliche Bildbestand von rechtshistorischer Seite her keine besondere Beachtung fand. ${ }^{14}$ Der Übergang der Handschrift in die Sammlung Ludwig und letztendlich in das Paul Getty-Museum in Malibu ${ }^{15}$ ließ das Interesse

\footnotetext{
${ }^{4}$ https://drw-www.adw.uni-heidelberg.de/drw-cgi/zeige?term=krieg\&index=lemmata (4. 5. 2020).

${ }^{5}$ Dieses „,rechtsförmliche Verfahren“ konnte unter Umständen in eine vom modernen Kriegsbegriff nicht

vollständig zu trennende „kampfliche Auseinandersetzung“ in Form der Fehde münden, vgl. https://drw-

www.adw.uni-heidelberg.de/drw-cgi/zeige?term=krieg\&index=lemmata (4. 5. 2020): III $1 \mathrm{~b}$.

${ }^{6}$ HERMANN, Friede 1807.

${ }^{7}$ HeRMANN, Friede 1810-1812.

${ }^{8}$ Hermann, Friede 1814. Vgl. dazu auch Kocher, Friede und Recht 405-415 (mit Illustrationen).

9 ANDERMANN, Das Recht im Bild 421-427. Vgl. auch den kurzen Abriß der Entwicklung der rechtsikonographischen Forschung mit einführender Literatur auf der kürzlich ins Netz gestellten Homepage unter: http://www.rechtsikonographie.de/.

${ }^{10}$ Etwa Schwabenspiegel, Belialillustrationen, romanistische und kanonistische illustrierte Handschriften.

11 Vgl. EBEL - FIJAL - KOCHER, Römisches Rechtsleben.

12 TILANDER, Vidal Mayor.

${ }^{13}$ KaUfFmanN, Vidal Mayor und EuW - PlotzeK, Handschriften.

${ }^{14}$ KAUfFMANN, Vidal Mayor 112-138 setzt sich nur mit den 11 großen Randminiaturen auseinander; EUW - PLOTZEK, Handschriften 63-77 beschreibt zwar alle 156 Ilustrationen, ohne sich allerdings in eine wirkliche rechtliche Interpretation einzulassen.

$15 \mathrm{Zu}$ den Vorbesitzern der Handschrift Euw - PLOTZEK, Handschriften 63 und zum Übergang an die GettySammlung in Malibu 1983 ebd. 11.
} 
an dieser bemerkenswerten Handschrift nicht erlahmen, sie wurde sogar zum Ziel eines von der Österreichischen Akademie der Wissenschaften geförderten Dissertationsprojektes. ${ }^{16}$ Ungeachtet dieser Tatsache ${ }^{17}$ und geleitet von dem durch den Übergang an die Getty-Sammlung ermöglichten freien digitalen Zugang zur Handschrift soll an Hand von 5 Beispielen aus dieser Handschrift die Visualisierung von „Krieg und Frieden“ im weitesten Sinn des Wortes belegt werden. Die Betrachtungsbogen reicht vom Vertrag als manifestiertem Frieden über den Streit („Krieg“) als auslösenden Faktor bis zu unterschiedlichen Formen der Wiederherstellung des Friedens; dazu gehört die Beilegung zwischen den Parteien ohne äußere Hilfe wie auch die Einschaltung neutraler friedensstiftender Institutionen (Gericht) oder von Personen mit hohem Sozialstatus.

\subsection{Die Begründung einer geordneten, friedlichen Rechtsbeziehung}

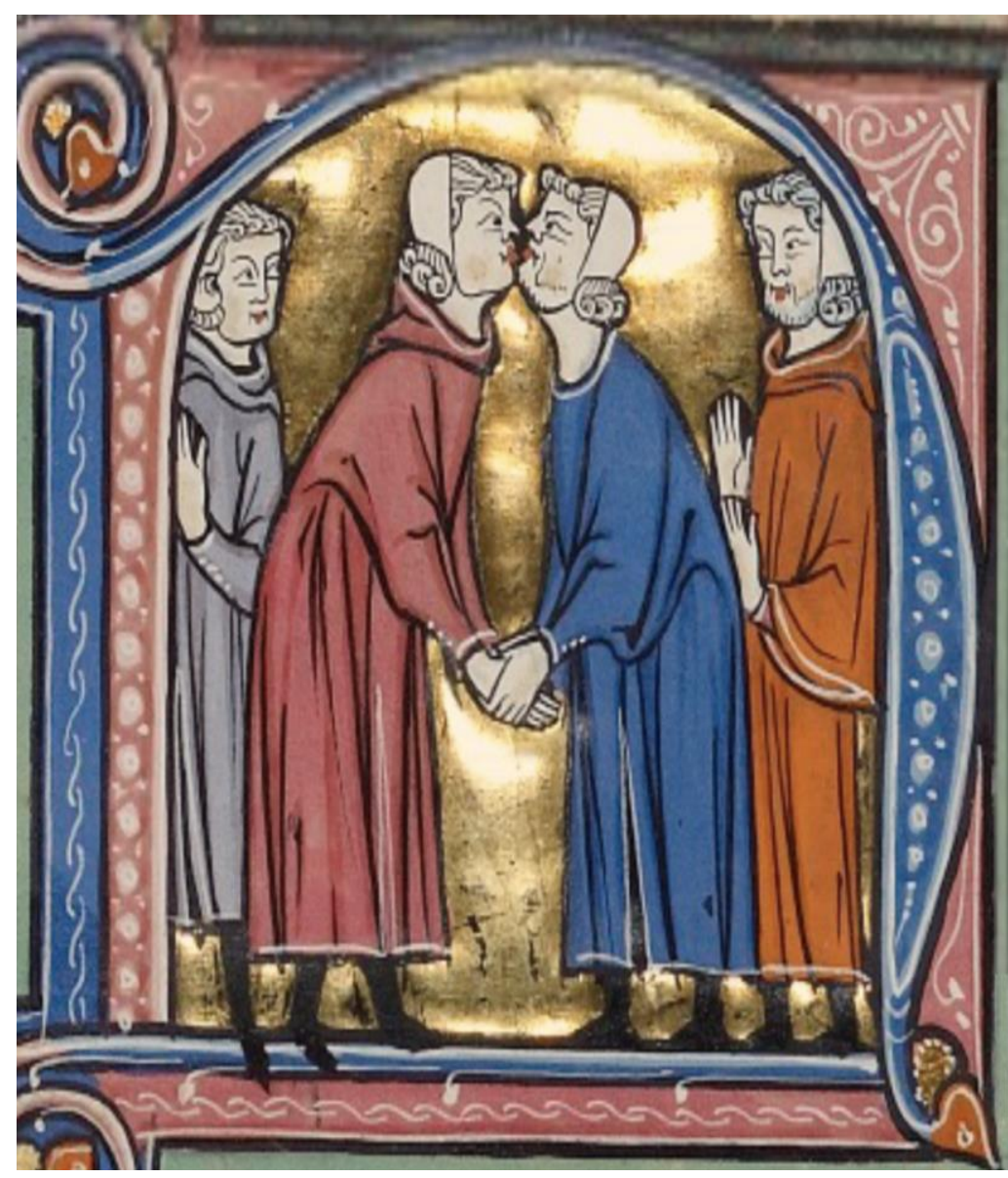

Eröffnet wird der Bilderzyklus mit einer Frieden und Ordnung repräsentierenden Darstellung aus dem siebenten Buch, das dem Militärwesen gewidmet ist. Grundelemente des mittelalterlichen Militärwesens sind die zu leistenden Dienste, ob nun Versorgung, Mauerbau oder aktiver Kampf-einsatz. Im nordspanischen Bereich hat sich dazu ein Rechtsinstitut entwickelt, das als Basis für die Sicherung dieser erforderlichen Dienstleistungen diente, das Homagium (aragonesisch: homenage) und das sich dann in der Folge über den europäischen Rechtsraum verbreitet hat. $^{18}$

Abbildung $1^{19}$

\footnotetext{
16 https://kunstgeschichte.univie.ac.at/forschungsprojekte/buchmalerei/vidal-mayor/ (11. 7. 2020).

${ }^{17}$ Und in Übereinstimmung mit Frau Maga. iur. Kristina Kogler BM MA in deren Händen die Durchführung dieses Projektes liegt. Sie hat sowohl eine kunsthistorische als auch juristische Ausbildung, was neben der primär kunsthistorische Einordnung sekundär auch auf eine Beleuchtung der rechtliche Seite abzielt.

${ }^{18}$ DiestelKaMP, Homagium 1116-1118.

19 http://www.getty.edu/art/collection/objects/4558/unknown-michael-lupi-de-candiu-initial-n-two-men-shakinghands-and-kissing-spanish-about-1290-1310/ (8. 6. 2020).
} 
Artikel 23 des siebenten Buches widmet sich ausführlich diesem Thema, wobei Absatz 3 die konstitutive Funktion des Homagium im Hinblick auf die Leistungspflicht feststellt. Die einleitende Illustration (Abbildung 1/fol. 230r) knüpft thematisch hier an, denn sie konzentriert sich auf die Begründung dieses Dienstverhältnisses durch einen entsprechenden Formalakt: Der Herr (rechts, in blauer Kleidung) steht dem künftigen Vasallen gegenüber und umschließt mit seinen Händen die gefalteten Hände des neuen Dienstmannes. Die leicht vorgeneigte Haltung beider mit den sich dadurch stark annähernden Gesichtern deutet den Lehenskuss an. Begleitet wird die Szene links und rechts je von einem Zeugen als Ausdruck der Öffentlichkeit, vor der ein rechtlich so bedeutsamer Akt über die Bühne ging. Dieser Formalakt findet sich auch in anderen juristischen und nichtjuristischen Handschriften in unterschiedlicher Ausgestaltung. ${ }^{20}$ Dass ein solcher Einigkeit und Ordnung ausdrückender Akt im Hintergrund auch wieder „Krieg“ bedeuten kann, liegt in der Folgepflicht des Vasallen bei militärischen Auseinandersetzungen begründet, kann aber auch bei einer Störung des Dienstverhältnisses selbst in gerichtliche oder kampfliche Auseinandersetzung münden. ${ }^{21}$

\subsection{Der Ärger mit den Leiharbeitern - Konfliktbeilegung ohne Gericht}

Die oben bei Abbildung 1 angedeutete Möglichkeit des Streites („Krieg“) wegen der Störung eines durch Vertrag geregelten rechtlichen Beziehungsverhältnisses wird hier im nichtmilitärischen Bereich im Rahmen einer locatio conductio, also eines Vertrages über die Bereitstellung eines Leiharbeiters konkretisiert. Die Verwandtschaft mit dem Homagium ist durchaus zu sehen, denn dort wie hier geht es um die Verpflichtung von Personen, die eine Leistung erbringen sollen. Die Beilegung des Streites erfolgt hier durch die Parteien selbst ohne die Bemühung einer externen Institution oder Person. Bei der Auswahl der Personen, die man als Vermittler anbietet, sollte man Sorgfalt walten lassen, um Streitigkeiten zu vermeiden. Wenn ein solcher Leiharbeiter vor der vereinbarten Zeit ohne Schuld des Dienstherrn oder ohne sonstigen ehrenhaften Grund seine Arbeitsstelle verläßt, so kann das noch ohne eine vermittelnde Institution (Gericht) saniert werden, sofern der Vermittler einen Ersatz für die restliche ausstehende Arbeitsverpflichtung beschafft.

Die Illustration zu Titel 7 des fünften Buches (Abbildung 2/fol. 172v) knüpft an dieses Szenario an. Dass die im Text erwähnte Störung der locatio conductio die Ausgangslage für das Bild war, ergibt sich aus der Tatsache, dass der blau gekleidete, größenmäßig hervorgehobene und im Bildzentrum stehende Stellenvermittler die rechte Hand hoch erhoben hat um klarzustellen, dass er seiner Ersatzpflicht nachkommt. Der Dienstherr bestätigt mit dem Zeigefinger der rechten Hand die Lösung des Problems. Der Übergang des Ersatzmannes aus der personenrechtlichen Gewalt des Vermittlers in jene des Dienstherrn erfolgt mit zwei Gebärden: Der Vermittler schiebt den Dienstboten mit der linken Hand in Richtung des Dienstherrn und dieser ergreift ihn mit seiner linken Hand an dessen rechten Handgelenk.

Ob die weiteren zwei Personen, die rechts eher im Hintergrund stehen, zum Vermittler gehören oder weitere Ersatzpersonen sind, muß offen bleiben. Für beide Varianten ist ein

${ }^{20}$ KOCHER, Zeichen und Symbole Abb. 2, 61, 190 sowie GARNIER, Le langage di l'image Nr. 169: Homagium zwischen dem König von Frankreich und dem König von Navarra, mit einfacher Handreichung und Kuss. Nur Handreichungen ebd., Nr. 88 und 168.

21 SPIESS - THEUERKAUF, Felonie 1534-1535. 


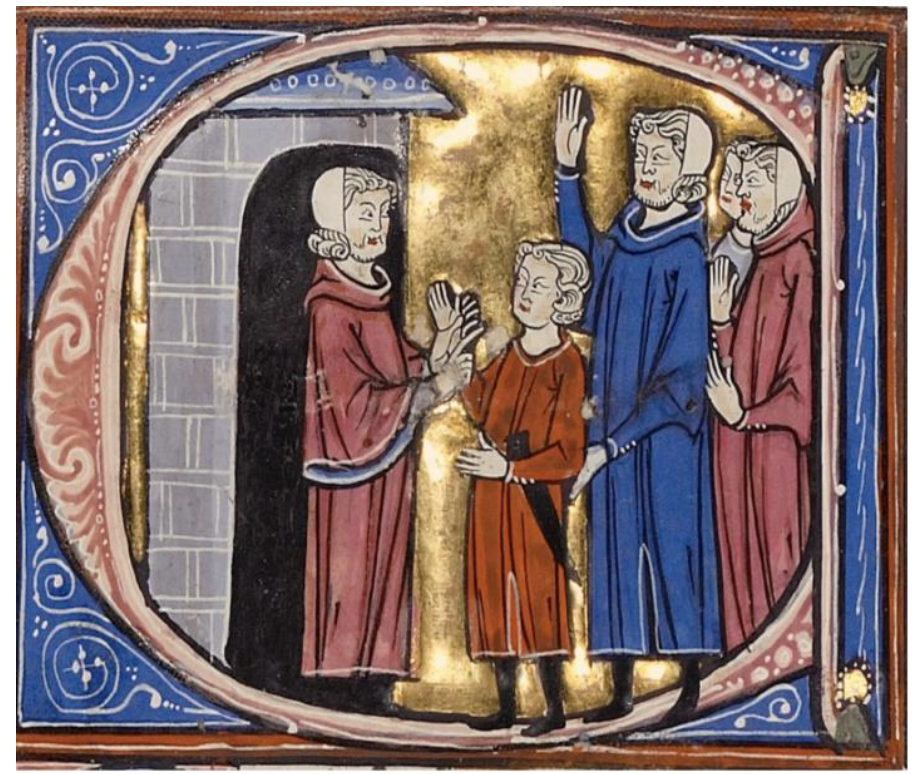

gebärdentechnischer Ansatzpunkt vorhanden, denn der rot gekleidete Mann hinter der dominierenden Vermittlerfigur bringt sich auch mit einer erhobenen Hand in das Geschehen ein. Der Künstler hat hier mit einem in der Bildsprache häufig verwendeten Muster gearbeitet, nämlich mit der Größe der Personen in Relation zu ihrer Bedeutung: Die Hauptperson, der Dienstvermittler, ist besonders groß, der sozial niedrig einzustufende und auch austauschbare Arbeiter ist verhältnismäßig klein.

Abbildung $2^{22}$

\subsection{Friedensbruch im geschützten Bereich - Sanierung durch Geldstrafe}

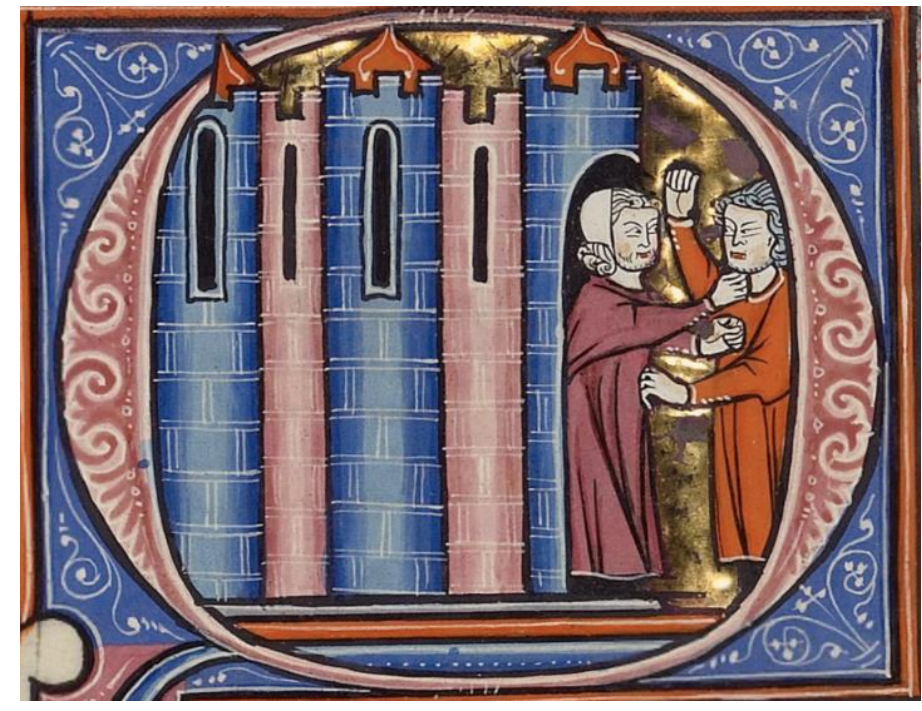

Das neunte Buch beschäftigt sich ausführlich mit dem Strafrecht, dessen Tatbestände und Strafen im Regelfall leichter zu visualisieren sind als andere Rechtsbereiche. Artikel IX, 48 beschäftigt sich überwiegend mit Grundtatbeständen und den darauf stehenden Strafen. Absatz zwei geht von einem Friedensbruch durch Gewalttat in einem geschützten Bereich, einem ummauerten oder zumindest irgendwie geschützten Ort, aus.

\section{Abbildung $3^{23}$}

Die Illustration (Abbildung 3/fol. 269r) bietet eine Stadtmauer und im Tor stehen zwei Männer, die aufeinander mit Fäusten losgehen. Die Szene ist als normale Schlägerei konzipiert und

\footnotetext{
22 http://www.getty.edu/art/collection/objects/4523/unknown-michael-lupi-de-candiu-initial-e-three-men-and-aboy-meeting-another-man-in-his-doorway-spanish-about-1290-1310/?dz=0.3883,0.2978,2.99 (8. 7. 2020).

${ }^{23}$ http://www.getty.edu/art/collection/objects/4588/unknown-michael-lupi-de-candiu-initial-q-a-man-greetingtwo-men-from-his-doorway-initial-q-two-men-fighting-in-front-of-a-house-spanish-about-1290-1310/ (8. 7. 2020 rechte Spalte).
} 
darauf wird eine Buße von 500 Solidi angedroht, die sich verdoppelt, wenn die Sache tödlich ausgeht; diese Variante wird allerdings nicht visualisiert. Hier ist der Illustrator beziehungsweise der Konzipient extrem sparsam gewesen, denn das wesentliche Element, die Strafbarkeit oder der Deliktscharakter kommt im Bild nicht zum Ausdruck - dabei wäre das durchaus machbar gewesen: Eine Zweiteilung des Bildes mit einer Folgeszene vor dem Richter hätte diese Informationslücke füllen können. Bei der Abbildung hat er sich dieser Lösung bedient, hier erschien es ihm wohl nicht wichtig genug.

\subsection{Der unfreundliche Nachbar - Friedensstiftung durch das Gericht}

Im vierten Beispiel begnügt man sich nicht mehr mit Wortgefechten, sondern schreitet zur Anwendung von körperlicher Gewalt.

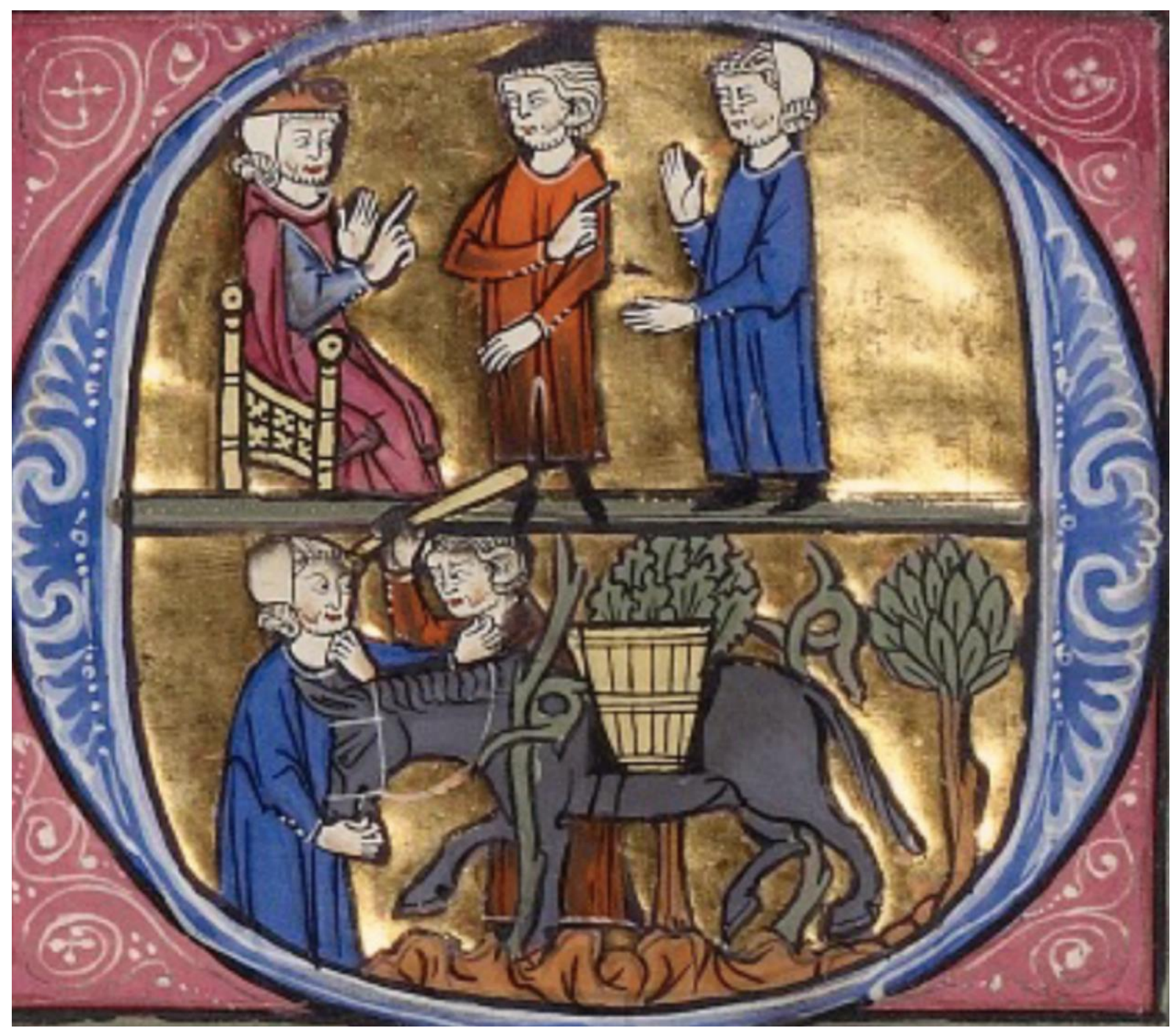

Abbildung $4^{24}$

24 http://www.getty.edu/art/collection/objects/4516/unknown-michael-lupi-de-candiu-initial-q-two-men-arguingtheir-cases-before-a-judge-and-men-with-a-donkey-spanish-about-1290-1310/?dz=0.6657,1.0542,1.27 (8. 7. 2020). 
Es handelt sich im Grund genommen um eine Alltagssituation: Jemand besitzt einen Weingarten, der keinen Zugang zu einem öffentlichen oder privaten Weg hat. Die Illustration zu Artikel 44 des vierten Buches behandelt das Problem in einer Zweierbildfolge (Abbildung 4/fol. 167v). Das untere Bild in der Initiale zeigt die Ausgangssituation: Der Eigentümer oder Pächter des Weingartens möchte die Ernte mit einem Esel notgedrungen über fremden Grund und Boden hinausbringen und der Eigentümer des fremden Weingartens hindert ihn mit Gewalt daran: Er packt ihn am Halsausschnitt seines Gewandes und schlägt ihm mit einem Knüppel auf den Kopf, der Angegriffen kann nur eine Abwehrbewegung mit der linken Hand machen. Im oberen Bild sieht man sich vor dem örtlich zuständigen Richter wieder: Der an seiner Notwegpassage Gehinderte hat Beschwerde erhoben (rechte Hand erhoben, offene Handfläche nach vorne gekehrt, linke Hand weist auf den Kontrahenten) und bekommt vom Richter, der mit seiner linken erhobenen Hand auf den Kläger eingeht, Recht. Der mit dem weisenden Zeigefinger der rechten Hand des Richters ausgedrückte Spruch zu Gunsten des Klägers wird vom Beklagten übernommen. Hervorzuheben ist, dass der Illustrator mit Hilfe der Kleiderfarbe Identitätsgleichheit ausdrückt, eine Methode, die in den Sachsenspiegelbildern konsequent genützt wird, während in den anderen illustrierten Rechtshandschriften diese Variante der Bildsprache kaum vorkommt. Der über das Verbale hinaus in körperlichen Angriff ausgeartete Friedensbruch wird durch den Spruch des lokalen Richters wieder saniert.

\subsection{Der König stellt den Frieden wieder her}

Die ersten beiden Artikel des achten Buches beschäftigen sich mit dem Problem der Aufrechterhaltung beziehungsweise der Wiederherstellung des inneren Friedens im Lande. Da die Friedenswahrung zu den wichtigen Aufgaben eines Herrschers zählt, ist es selbstverständlich, dass in beiden Illustrationen der König im Mittelpunkt steht. Während er aber in der Illustration zu VIII,4 (fol. 237v - hier nicht wiedergegeben ${ }^{25}$ ) in klarer Machtposition (mit der Krone auf dem Haupt, auf dem Thron sitzend, das Schwert in der linken Hand und mit Befehlsgebärde der rechten Hand) dargestellt ist, findet er sich in der hier wiedergegebenen Miniatur zu VIII,2 (Abbildung 5/fol. 237r) wohl mit der Krone auf dem Haupt als König ausgewiesen, doch er befindet sich in einer reinen Vermittlerposition, indem er im Begriff ist, die Hände der beiden Anführer der Streitparteien zusammenzuführen. Beim Modus dieser Streitbeilegung ergibt sich eine Differenz zum Text, denn dort ist die Rede von mündlichen oder schriftlichen Weisungen, während hier die Gebärdensprache zum Tragen kommt. Es ist also eine stark vereinfachte Darstellung, die mit dem Text nur die Tatsache der Friedensstiftung gemein hat, während zusätzlich die politische Realität im Bild fehlt, nämlich die Bezugnahme auf die Mitwirkung der Stände sowie des bischöflichen Rates und des königlichen Gerichtshofes. Die Androhung mit königlicher Macht bei weiterem Friedensbruch gegen die ungehorsame Partei eingreifen zu wollen, ist ebenfalls visuell nicht präsent. Auch die hier nicht wiedergegebene Miniatur zu VIII, ${ }^{26}$ läßt diesen politischen Mitwirkungsaspekt beiseite und begnügt sich mit zwei Adeligen, die sich - auf königlichen Befehl

\footnotetext{
${ }^{25}$ http:/ / www.getty.edu/art/collection/objects/4565/unknown-michael-lupi-de-candiu-initial-e-four-men-before-a-
} king-spanish-about-1290-1310/ (8. 7. 2020).

26 Wie Anm. 25. 
hin - die Hand reichen. Trotz der großen Differenz zwischen Bild und Text ist die friedwahrende Funktion des Herrschers klar ablesbar. Unter Umständen könnte man auch die eindeutig auf den Herrscher weisenden Hände der beiden Parteien als vom Künstler gewollte Demonstration der Machtposition des Herrschers ${ }^{27}$ auffassen, worin dann auch eine Relativierung der Bedeutung der politischen Kräfte gesehen werden könnte.

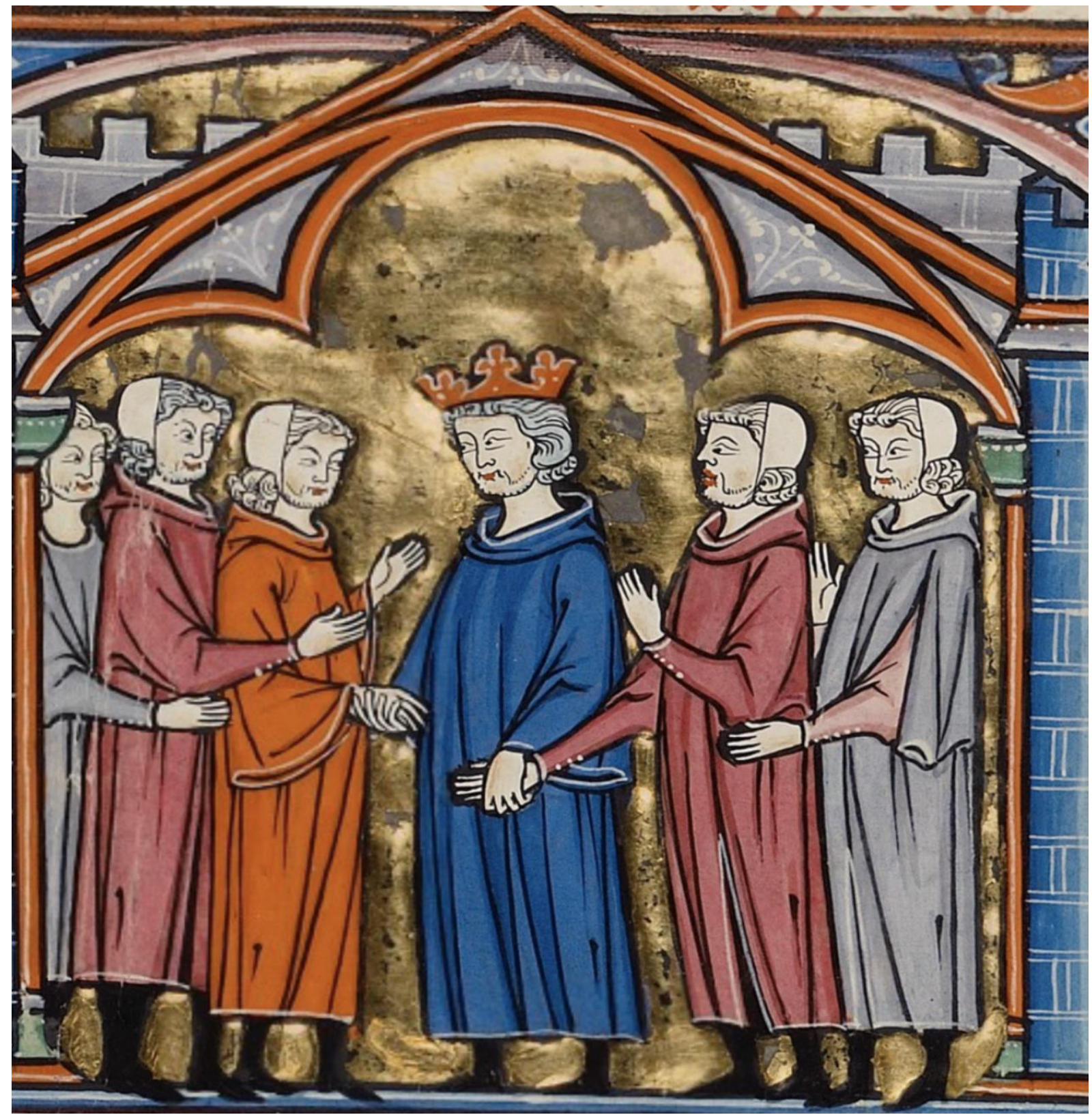

Abbildung $5^{28}$

\footnotetext{
27 WiTTEKIND, Der König als Gesetzgeber und Rechtsgarant 138-167.

2828 http:/ / www.getty.edu/art/collection/objects/4564/unknown-michael-lupi-de-candiu-initial-e-a-king-and-fivemen-spanish-about-1290-1310/ (8. 7. 2020).
} 


\section{Ausblick}

Der Vidal Mayor umfasst insgesamt 156 Illustrationen, daran gemessen bieten die 5 Buchmalereien nur einen minimalen Einblick in das Leistungsspektrum von Bildanweiser und Künstler. Diese „Momentaufnahmen“ über Teile rechtlicher Sachverhalte und Regeln können aber sehr gut zeigen, dass die Wortvielfalt zu „Krieg und Frieden“ durchaus eine Entsprechung im visuellen Bereich hat. Körpersprache kann Einigkeit und Frieden genauso zum Ausdruck bringen, wie Streit („Krieg“) und in der Folge Wiederherstellung des Friedens.

\section{Literatur- und Quellenverzeichnis}

ANDERMANN, Ulrich: Das Recht im Bild. Vom Nutzen und Erkenntniswert einer historischen Quellengattung (Ein Forschungsüberblick). In: LÖTHER, Andrea - MEIDER, Ulrich - SCHNITZLER, Norbert et al. (Hrsg.): Mundus in imagine. Bildersprache und Lebenswelten im Mittelalter. Festgabe für Klaus Schreiner. Mit einem Geleitwort von Reinhard Koselleck. München 1996, 421-451.

CORDES, Albrecht - HAFERKAMP, Hans-Peter - LÜCK, Heiner et al. (Hrsg.): Handwörterbuch zur deutschen Rechtsgeschichte $\mathrm{III}^{2}$. Berlin 2016, 251.

DiestelKAMP, Bernhard: CORDES, Albrecht - HAFERKAMP, Hans-Peter - LÜCK, Heiner et al. (Hrsg.): Homagium. Handwörterbuch zur deutschen Rechtsgeschichte II². Berlin 2012, 1116-1118.

EBEL, Friedrich - FIJAL, ANDREAS - KOCHER, Gernot: Römisches Rechtsleben im Mittelalter. Miniaturen aus den Handschriften des Corpus iuris civilis. Heidelberg 1988

Euw, Anton von - PlotzeK, Joachim M.: Die Handschriften der Sammlung Ludwig, Band 4. Köln 1985

GARNIER, Francois: Le langage di l'image au moyen age. Signification et symbolique. Paris 1982

Herger, Eszter Cs. - Korsósné DelaCAsSE, Krisztina et al. (Hrsg.): Recht ohne Grenzen. Festschrift zum 15. Jubiläum der Zusammenarbeit der Grazer und Pécser Rechtshistoriker. Pécs 2007

Hermann, Hans-Georg: Friede. In: CORDES, Albrecht - HAFERKAMP, Hans-Peter - LÜCK, Heiner et al. (Hrsg.): Handwörterbuch zur deutschen Rechtsgeschichte I². Berlin 2008, 1807-1821.

https://drw-www.adw.uni-heidelberg.de/drw-cgi/zeige?term=krieg\&index=lemmata (4. 5. 2020)

https://drw-www.adw.uni-heidelberg.de/drw-cgi/zeige?term=krieg\&index=lemmata (4. 5. 2020): III 1 b.

https://kunstgeschichte.univie.ac.at/forschungsprojekte/buchmalerei/vidal-mayor/ (11. 7. 2020).

http://www.getty.edu/art/collection/objects/4565/unknown-michael-lupi-de-candie-initial-e-four-men-before-a king-spanish-about-1290-1310 (8. 7. 2020)

http://www.getty.edu/art/collection/objects/4564/unknown-michael-lupi-de-candiu-initial-e-a-king-and-five-menspanish-about-1290-1310/ (8. 7. 2020)

http://www.getty.edu/art/collection/objects/4516/unknown-michael-lupi-de-candiu-initial-q-two-men-arguingtheir-cases-before-a-judge-and-men-with-a-donkey-spanish-about-1290-1310/?dz=0.6657,1.0542,1.27 (8. 7. 2020)

http://www.getty.edu/art/collection/objects/4588/unknown-michael-lupi-de-candiu-initial-q-a-man-greeting-two men-from-his-doorway-initial-q-two-men-fighting-in-front-of-a-house-spanish-about-1290-1310/ (8. 7. 2020 rechte Spalte)

http://www.getty.edu/art/collection/objects/4523/unknown-michael-lupi-de-candiu-initial-e-three-men-and-a-boya nother-man-in-his-doorway-spanish-about-1290-1310/?dz=0.3883,0.2978,2.99 (8. 7. 2020)

http://www.getty.edu/art/collection/objects/4558/unknown-michael-lupi-de-candiu-initial-n-two-men-shakinghands-and-kissing-spanish-about-1290-1310/ (8. 7. 2020)

http://www.rechtsikonographie.de/ (8. 7. 2020) 
KAJTÁR, István: Bevezetés a jogi kultúrtörténetbe [Einführung in die rechtliche Kulturgeschichte]. Budapest - Pécs 2004

KaufFmanN, Carl Michael: Vidal Mayor: Ein spanisches Gesetzbuch aus dem 13. Jahrhundert in Aachener Privatbesitz. Aachener Kunstblätter 29, 1964, 108-138.

KOCHER, Gernot: Friede und Recht. In: HAuCK, Karl - KroeSCHELl, Karl - SONDEREGGER, Stefan et al. (Hrsg.): Sprache und Recht. Beiträge zur Kulturgeschichte des Mittelalters. Festschrift für Ruth Schmidt-Wiegand zum 60. Geburtstag. Berlin - New York 1986, 405-415.

KOCHER, Gernot: Zeichen und Symbole des Rechts. Eine historische Ikonographie. München 1992

MÜNKLER, Herfried: Krieg. In: CORDES, Albrecht - HAFERKAMP, Hans-Peter - LÜCK, Heiner et al. (Hrsg.):

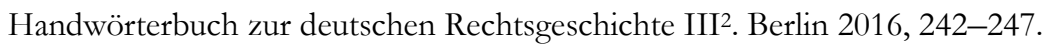

SPIESS, Karl-Heinz - THeuerkAuf, Gerhard: Felonie. In: CORDES, Albrecht - HAFERKAMP, Hans-Peter - LÜCK, Heiner et al. (Hrsg.): Handwörterbuch zur deutschen Rechtsgeschichte I². Berlin 2008, Sp. 1534-1535.

Tilander, Gunnar: Vidal Mayor. Traducción aragonesa de la obra In excelsis Dei thesauris de Vidal de Canellas, 3 Bände. Lund 1956

WitTEKIND, Susanne: Der König als Gesetzgeber und Rechtsgarant in den Miniaturen des Libro de las Leyes (London, British Library, Add. Ms. 20787). In: BÖSE, Kristin - WITTEKIND, Susanne (Hrsg.): AusBILDung des Rechts. Systematisierung und Vermittlung von Wissen in mittelalterlichen Rechtshandschriften. Frankfurt 2009, 138-167. 